\title{
Autonomous Organization of Wireless Network Transport in a Multi-Provider Environment
}

\author{
Lester T. W. Ho ${ }^{1}$, Francis J. Mullany ${ }^{2}$, Holger Claussen ${ }^{1}$, Louis G. Samuel ${ }^{1}$ \\ ${ }^{I}$ Wireless Research Lab, Bell Labs, Lucent Technologies, The Quadrant, Stonehill Green, Swindon, United Kingdom. \\ ${ }^{2}$ Bell Labs Ireland, Lucent Technologies, Blanchardstown Industrial Park, Dublin 15, Ireland.
}

\{lho1, mullany, claussen, lsamuel\}@lucent.com

\begin{abstract}
With the emergence of multiple wireless operators using a variety of access technologies, congested spectrum bands, and multi-standard user terminals, there is a pressing need to see what implications autonomic communication systems can have on business models in the wireless access business. This paper outlines and discusses some perspectives on the future impact of autonomicity in a demand-, resource-, and competitiondriven environment and on the strategies of wireless access providers. The effects are investigated for a standards-neutral, multi-agent architecture that is capable of per-session/call trading of access to wireless network and spectrum resources, exploiting the capability of autonomous agents to represent the business interests of individual players. Results from a joint radio-resource/inter-agent-negotiation simulator are used to quantitatively assess the economic outcomes in various multi-operator competitive scenarios in a realistic environment (an international airpor.t)
\end{abstract}

\section{Introduction}

System architectures employing a multiplicity of autonomous agents have been proposed for various applications in the communications domain [1]. However, it is often unclear as to where multi-agent systems provide such a clear advantage over conventional techniques that they will be actually implemented and exploited in real-life contexts. The aim of this paper is to explore this issue, using a particular agent architecture discussed in Section 2, in the application domain of wireless network transport provision. Based on previous work [2][3], the architecture allows different, autonomous agents to represent the various business players such as the end user, brokers, and a multiplicity of operators/network providers. It enables them to trade in transport provision at granularities as fine as individual sessions or calls. The question addressed by the rest of the paper is the viability of this arrangement.

\footnotetext{
${ }^{1}$ This work was part-supported by the EU Commission through the IST-2001-39117 IST FP5 project ADAMANT.
}

As is made clear by planned future research directions [4], there are likely to be further major changes in the delivery mechanisms for the communication services of the future: the basic wireless network architectures will move further away from the centralized, hierarchical, functionally layered networks of today to distributed, flatter access networks with simpler IPbased core networks, as shown in a very simplified form in Figure 1. The implications of such a "network of networks" [5] and the attendant fragmentation of the market for network transport supply are explored qualitatively in Section 3. It is shown that the proposed agent architecture has some significant advantages for all key business players in such an environment.

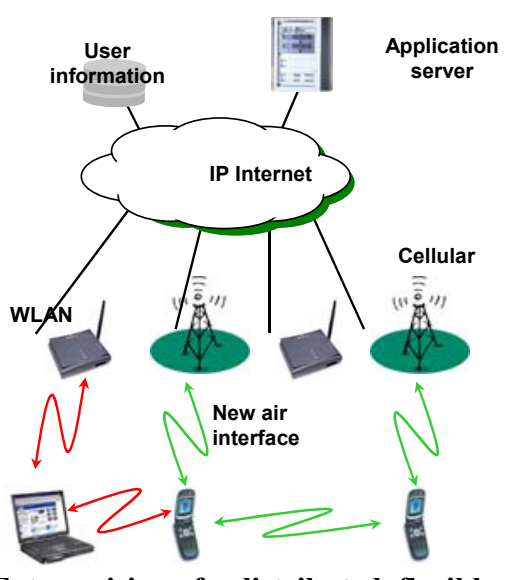

Figure 1. Future vision of a distributed, flexible wireless access network

However, qualitative statements are not sufficient to assess the economic viability of any new proposal: as a result, Section 4 describes a simulator that captures the key features of (a) the agent architecture and inter-agent communications, (b) the agent strategies, (c) the user demand for communication services, and (d) the supply of radio resources and access equipment such as base stations. Economic modelling of the various players is an important part of the simulator, allowing the assessment of the market outcomes in various multi-operator competitive scenarios in a realistic environment (an international airport), as shown in Section 5. Some conclusions are given in Section 6. 


\section{An Autonomic Agent-based Architecture}

Figure 2 shows the proposed agent architecture as a controlling layer superimposed on the traditional elements (shown in a very simplistic manner) in a communications system. Agents, in this context, are autonomous software entities capable of acting on behalf of agendas that are not necessarily shared by other agents.

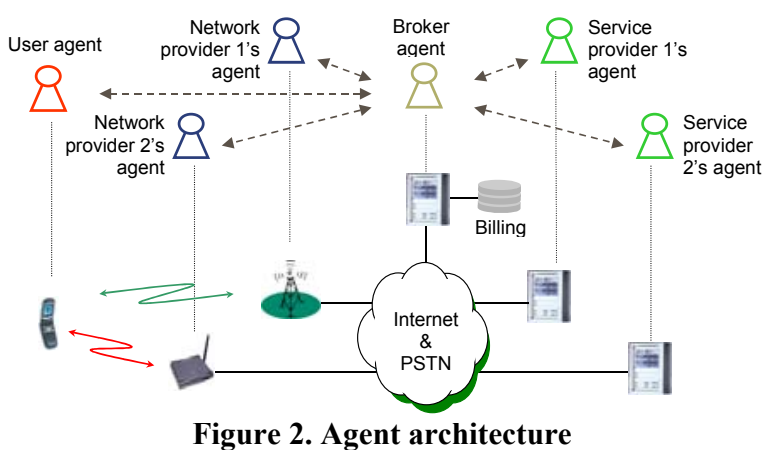

It is this independence that is critical to the concept of an agent: it enables each agent to act on behalf a different business entity, representing the technical/economic interests of that entity. How the agents act, the decisions it makes, and the strategy it follows is up to the agent itself. This is different from other computing paradigms, in that while it is possible for a monolithic system to act on behalf of various users (e.g. an internet-based stock brokerage service that allows customers to set buy and sell thresholds for given stocks), the actions taken by monolithic systems are generally inflexible and the strategies simplistic.

Thus, autonomous agents allow one to break away from the current, fixed business relationships between providers and end-users (e.g. post-pay cellular subscription services). As shown in Figure 2, a number of different agents are used, each acting on behalf of the business entity for which it is named, namely (a) user agents (each for one individual user only), (b) network provider agents, (c) a broker agent, and (d) service provider agents. Here, the decision was made to partition service provision from network transport provision since today's architectures allow this. For example, in R5 UMTS, one has a partitioning between IMS and the core and radio access networks. Thus, the architecture, from a business perspective, has the maximum flexibility implied by the technology, even if in some scenarios this flexibility is not exploited. One can envisage a range of market structures enabled by this architecture, ranging from the monolithic and monopolistic to the fragmented and competitive.
It is the agent architecture that organizes the underlying systems to provide an end-to-end communications services to the end-user. When the user requests a service (e.g. a voice call, data session, etc.), the user agent approaches the broker, which then negotiates with the service provider agents for the service requested and the network provider agents for the QoS required for the transport pipe needed to carry that service. This inter-agent negotiation can be triggered every time that a user requests a new session or less frequently, depending on the existence or non-existence of prior business arrangements between the various entities involved. Clearly, per-session negotiation of a contract or servicelevel agreement (SLA) between the end user and providers is a novel feature of this architecture and has to be economically and quantitatively justified for any given market structure.

The other important characteristic of the architecture is its independence of the underlying technology used to provide transport and services. Once the negotiation between the various entities is completed, the underlying infrastructure is allowed to operate in its usual, standardspecific manner. This agnosticism towards the infrastructure allows the agent architecture to interface with a multiplicity of heterogeneous networks, with different technologies. (Indeed, this overlay approach is the method currently deployed to integrate end-user access to WLAN and cellular systems.) An alternative approach to implementing the agent architecture above is to employ agents that either reside in or communicate with a 3GPP/3GGP2 IMS system [7]. There, the possibility of multiple networks communicating with one IMS system is inherently part of the architecture.

In short, the fundamental innovative features of the above autonomous agent architecture are (a) an ability to be mapped to various market arrangements with different business models, (b) enabling per-session trading of access to services and network transport and (c) agnosticism to the underlying technology providing the transport and services.

\section{Future Scenarios}

One can fairly ask why the network operators of today would consider the major business model changes implied by some of characteristics of the above architecture. To answer this, consider the changing environment for the main "assets" that a telecommunications operator exploits (and needs) to generate revenue:

(i) Service and application provision. It is still not clear what will be the "killer application" that will generate significant high-margin revenue from the broadband wireless access systems now being deployed. The fastest method for finding such an application is to allow a competitive "jungle" of 
service providers to compete. The business models behind NTT DoCoMo's very successful i-mode system in Japan [8] employed such a technique.

(ii) Network access infrastructure. While there is critical added value to be achieved with near complete radio coverage (i.e. network externality), the emergence of a variety of cheap, fast air interfaces may result in much traffic being siphoned off by a variety of access methods.

(iii)Spectrum. Whether licensed or unlicensed, spectrum for public commercial communication services is a rare commodity and various governmental telecommunications regulators are now recognizing that the fixed-use, untradable spectrum rights that they current dispense are not the most efficient use of this valuable commodity. They are now moving towards spectrum trading [9], opportunistic spectrum exploitation [10], and flexible usage terms [9].

(iv)Customer interface and billing. Customers want seamless, universal service and simple transparent billing. Many operators will wish to retain control over the relationship with the end-user.

Thus, of the four assets given above, three are likely to experience extreme fragmentation over the course of the next decade or so. The last asset (iv) is the one that end-users will demand remain consolidated and simple to use. Therefore, one can conclude that in the architecture of Figure 2, the natural role for today's operators is that of the broker. Other players are then free to specialize as service or network access providers.

For example, by integrating a number of network access providers into the system, each either specializing in providing coverage under particular circumstances (e.g. urban hot-spot, motorway/railway corridors, rural) or competing directly, the broker can achieve more complete coverage than would be economically possible by a single operator. Similarly, an agent architecture (although not the one shown in Figure 2 without some modification) could be used to trade in spectrum blocks whenever a given network provider had more traffic than its current spectrum allocation allowed. By allowing "division of labor," the economic efficiency of the telecommunications industry would increase, a key goal of many regulators.

Of course, a fully competitive environment is not always necessary and one could envisage a number of difference scenarios that are compatible with the above architecture:

(i) Business players taking on multiple roles. For example, the i-mode business model would have the network provision and broker roles played by the network operator.

(ii) Collaborative scenarios. One could envisage that a cellular network operator would use such a system to integrate multiple, small, disparate WLAN operators scattered throughout a region into a single unified offering. Since, again, the cellular operator would act as both broker and network provider, a collaborative arrangement may be more appropriate than a competitive one. (iii) Granularity of SLA negotiation may be larger than a single session. With current technology, the fastest feasible negotiation rate is that on a per-session basis. However, there is no reason why the architecture could not be used for the negotiation of more long-lasting contracts. Negotiation for spectrum rights would be a good example of this, where the level of spectrum required depends on the slower variation of the average aggregate load.

(iv) Only some sections of the architecture are exploited: It may be the case that users will prove resistant to the concept of per-session contracts, with the uncertainty in the pricing that this can imply. One alternative is that the users have a fixed contract with the broker and per-session negotiation only occurs between the providers and the broker. The broker then hedges against price variations using its long-term subscription policies with end-users.

Thus, there is great flexibility in the architecture achieved through the use of autonomous agents - the challenge is to identify those business models and technology combinations that make sense in the real world. The rest of this paper is concerned with such qualitative assessments for a limited number of scenarios concerning network transport provision. (Service provision could also be assessed using the same framework, but the concentration here remains on the more complex issues arising from the interaction between demand and radio resources in network transport provision.)

\section{Simulator}

The simulation model was implemented in $\mathrm{OPNET}^{\circledR}$, and captures the behavior of the various components of the system, from the users and software agents to the underlying radio resources of the networks. The agent architecture modeled is composed of the user agents (UA), broker agents (BA) and network provider agents (NPA). The service provider agents are not modeled as only the trading of network transport provision is investigated.

\subsection{Inter-agent interactions}

The trading of network provision is implemented using an auctioning mechanism. The auction type chosen is a simultaneous, sealed bid auction where the participating network providers place their bids simultaneously to the broker, and the broker chooses the winning bid based on price. The bids are kept secret from other bidders, as is the identity of the auction winner and the number of participants. The only feedback that a network provider agent receives is whether it has won the auction or not. Figure 3 shows the signaling used in the implementation of the auction within the agent architecture.

This auctioning mechanism was chosen over an open, sequential auction because (a) it is anticipated that network providers would not want their pricing strategies to be 
revealed to competitors, (b) it involves less signaling and (c) for a single unit auction, most standard auctions (whether they are simultaneous or sequential) have been shown to be revenue equivalent anyway [11].

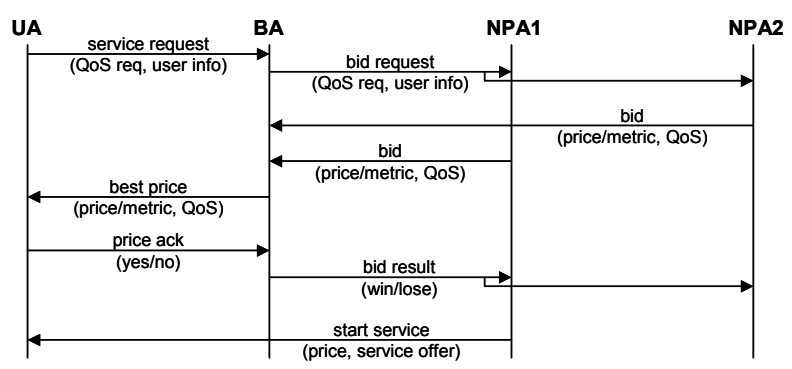

Figure 3. Signaling in an auction during a service request

\subsection{Demand and Resource Modeling}

The user demand was based on predictions of user behavior made in [12]. The users are divided into two types: business and casual users, each having different usage levels for each application. There are 11 applications available to the users, ranging from those requiring low QoS (e.g. SMS and email pings) to high QoS (e.g. streaming video and MP3 downloads).

Another aspect of user behavior that needs to be modeled is the user's willingness-to-pay threshold. This is the maximum amount in which the user will pay for a session, above which the user will reject the quote that it is offered. We assume that an individual user's threshold varies according to a normal distribution.

The radio resource simulator models the uplink and downlink for mobiles. It provides the required transmit power and spreading code resources for each link dependent on the positions, requested data rate, current interference from other base stations or mobiles, and channel conditions. In addition, the simulator provides functionality for call admission control and handover.

For this investigation, the base station maximum transmit power is considered to be $0.25 \mathrm{~W}$. A gain (antenna gain - cable loss) of $5 \mathrm{~dB}$ and a noise figure of $7 \mathrm{~dB}$ are assumed at the base station. At the mobile, the maximum transmit power is $0.125 \mathrm{~W}$, the gain is $0 \mathrm{~dB}$ and the noise figure is $9 \mathrm{~dB}$. The path loss is modeled as $L_{\mathrm{p}}=37+30 \log _{10}(d)$ using an implicit wall model, where $d$ is the distance in meters [13]. Finally, the shadowing attenuation is log-normal distributed with zero mean and standard deviation $12 \mathrm{~dB}$ [14]. For all shadow fading values, a correlation of 0.5 is assumed across all base stations.

\section{UMTS Results in Airport Environment}

The model is used to simulate the network in the airport environment of Athens International Airport (AIA). The system setup for this scenario has one broker and two competing network providers. The two network providers both operate UMTS networks. To differentiate the two network providers, one will have a single base station and the other two. Hence, one will have lower costs (but lower capacity) than the other. This differentiation is deliberate, as two competing networks with similar costs and network capacities in this market driven environment will only result in a load balancing effect, with users being distributed evenly between the two. Figure 4 shows the location of the base stations of

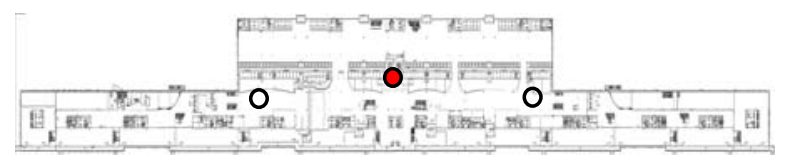

both providers within the terminal building.

Figure 4. Location of base stations within the airport

The passenger traffic levels were derived from actual passenger statistics of AIA and an assumed passenger take-up rate of $20 \%$ for the system, resulting in a traffic level of 400 users per hour. The ratio of business to casual users is assumed to be 1:4. The users are positioned randomly around the terminal and remain static throughout the simulations. The mean user's willingness to pay threshold is assumed to be $\$ 1.00$, with a variance of $\$ 0.01$.

\subsection{Network provider agent bidding strategies}

To study the effect of a per-session market driven scenario, the behavior of the network provider agents must also be modeled to reflect their likely pricing strategies, as this is one of the main factors that influences the system as a whole. The aim of the network provider agents when implementing a bidding strategy is to maximize its revenue, regardless of how optimally the network is used from a capacity point.

With this in mind, a suite of bidding algorithms was developed and implemented within the network provider agents. Using the limited amount of information on the auction outcome given by the broker agent, the bidding algorithms rely on the collected history of results of bids to approximate the effect of a particular bid level. This is done by participating in auctions with alternating trial bids, $b_{0}$ and $b_{0}+\Delta b$, and monitoring the probabilities, $p\left(b_{0}\right)$ and $p\left(b_{0}+\Delta b\right)$ of the bids winning for both cases. The steps for the bidding algorithm are as follows: i. Set $b_{\text {old }}=b_{0}$. 
ii. Partake in $n$ trial auctions for bids $b=b_{0}$ and

$$
b=b_{0}+\Delta b \text {. }
$$

iii. Measure the probability of success $p\left(b_{0}\right)$ and

$$
p\left(b_{0}+\Delta b\right) \text {. }
$$

iv. If $\left(b_{0}+\Delta b\right) \cdot p\left(b_{0}+\Delta b\right) \geq b_{0} \cdot p\left(b_{0}\right)$, set

$$
b_{0}=b_{0}+\Delta b \text {, else set } b_{0}=b_{0}-\Delta b \text {. }
$$

To maintain the credibility of the statistics collected during the trials, the $90 \%$ confidence interval of $p(b) . b$,

$t_{n-1,0.95} \sqrt{\frac{S^{2}(n)}{n}}$, is calculated after intervals of 50 trials, where $t_{n-1,0.95}$ is the upper 0.95 critical point for the $t$ distribution with $n-1$ degrees of freedom [15]. If the interval is smaller than a defined threshold, then the result is accepted and the trials stopped. Otherwise, the number of trials, $n$, is extended until the confidence intervals become small enough.

A network provider will never bid below its session cost price, as this would incur a loss. The algorithm tries to home in on the optimum bid value $b_{\text {opt }}$ value where $b_{\text {opt }}=\arg \max _{b}\{p(b) \cdot b\}$. All network providers would initially start bidding at cost, using $p(b)$ in their bidding algorithm.

\subsection{Results}

Figure 5 shows the resulting bids put in by the two competing network providers, $A$ and $B$, against time. The resulting bids that are put in by $B$ (the costlier operator) shows a tendency towards the approximate optimum bid price of $\$ 0.85$. Network provider $A$ (the cheaper operator) starts to display an undercutting strategy, where it will tend to bid just below its competitor. This setup is identical to what is known as a Bertrand model of competition, where each supplier announces the prices he intends to charge, and then customers buy services with preference for low prices [16]. In a Bertrand model game, the optimum bid (Nash equilibrium point) for the lower cost player is $b_{\text {lowcost }}=b_{\text {hicost }}-\varepsilon$, for infinitesimally small $\varepsilon$ [16], and this is reflected in the strategy adopted by $A$. Figure 6 shows the accumulated revenue of $A$ and $B$ over time, and it shows $A$ achieving a higher rate of revenue income compared to $B$, as expected.

It was found that the sessions that are won by $B$ came mostly from sessions that were not taken up by $A$ due to lack of capacity. From this, it is obvious that in a low demand environment such as a rural area, $B$ would not be able to obtain any revenue and this would result in monopolies existing in such areas. This also implies that network providers would have to place a much higher importance on costs when planning a network. It is now no longer desirable to have over provisioning, but rather, have just enough (or possibly less) capacity to satisfy demand at minimal cost.

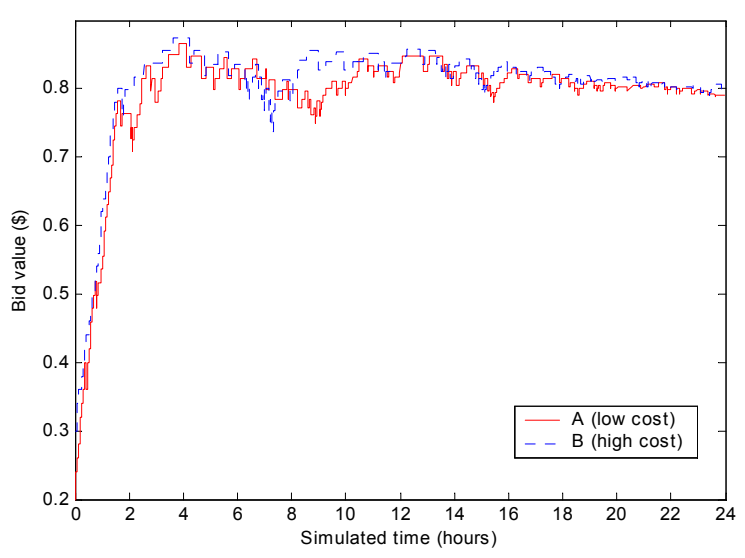

Figure 5. Value of bids placed by network providers

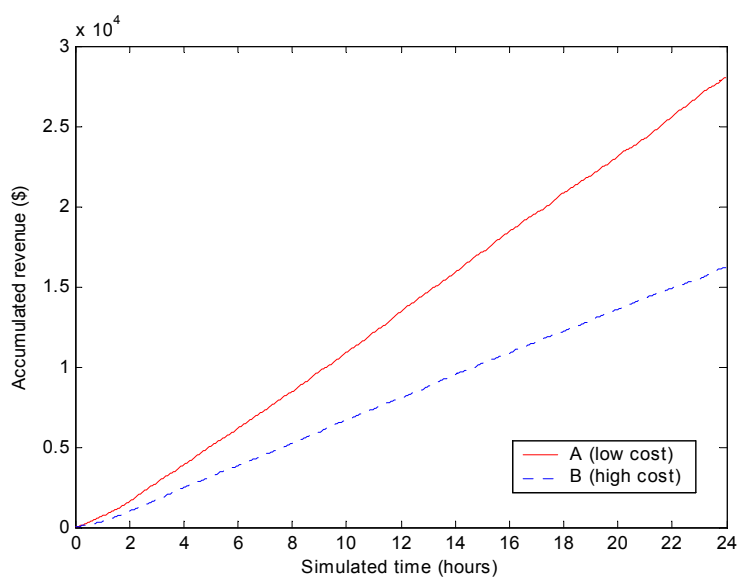

Figure 6. Accumulated revenue of network providers

The results shown so far have assumed that the network providers do not consider capacity when calculating bids. Figure 7 shows the accumulated revenue for $A$ and $B$ when capacity-linked bidding is implemented. This additional step adds a surcharge on top of the base bid $b_{0}$ (that was obtained using the bidding algorithm) whenever the session requested by the user will take up more than $2 \%$ of the available resources of a base station. The surcharge added on is proportional to the amount of capacity above the minimum $2 \%$ (e.g. $4 \%$ of capacity is charged two times $b_{0}, 6 \%$ three times, and so on). Figure 7 shows that $A$ achieves similar amounts of revenue as non-capacity linked bidding, but using only $31 \%$ of network resources, compared with $92 \%$ previously. $B$ 's revenue, however, decreases by a significant $90 \%$. A better option for $B$ is never to use capacity linked bidding, whereupon its revenue would improve, but revenue would still decrease by more than half after $A$ chooses to use capacity linked bidding. 


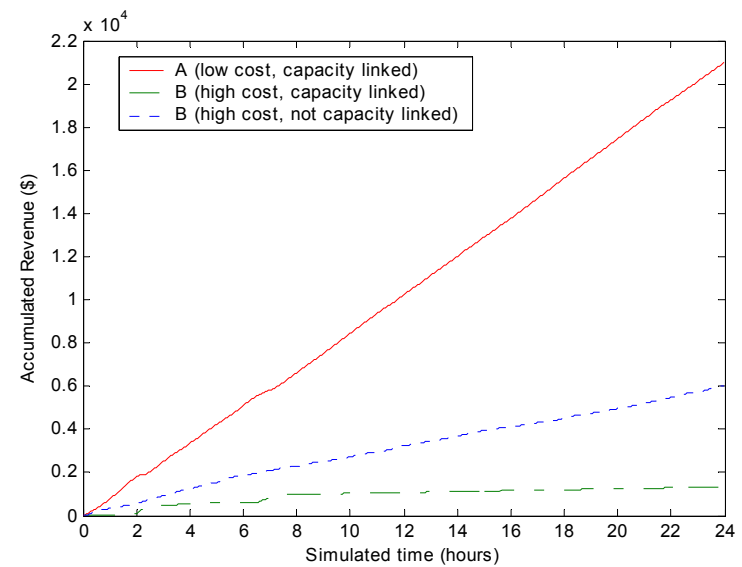

Figure 7. Accumulated revenue when capacity linked bidding is used

The introduction of capacity linked bidding shows the effect of network providers acting purely to supply network transport. Network transport would now be treated as a commodity and charged according to the amount used. This would cause high QoS applications such as video calling to become prohibitively expensive. Cross subsidization of high QoS applications would now be performed not by network providers, but the service providers.

\section{Conclusions}

This paper has outlined and discussed how system architectures employing autonomous software agents could be used in a future competition-driven environment, tying together multiple wireless operators using different access technologies to provide users with services that are standards agnostic via multi-standard terminals. In the future, the likely fragmentation of the various network operator assets into separate business entities will require radically new business models, moving from the current long term fixed user subscription to a more market-driven, flexible short term user relationship. An agent architecture, incorporating agents that represented each entity of this new fragmented market was put forward.

The effects of this market-driven structure was investigated for a standards-neutral, multi-agent architecture on a per-session trading for network transport. The results show that network providers now have more flexibility in maximizing their revenue. They are now able to disregard less profitable sessions for those with higher profit margins. Over-provisioning of network capacity is also no longer desirable. Network providers would also regard network transport as a commodity. As a result, cross subsidization of high QoS applications would be made at the service level, rather than the transport level.

\section{Acknowledgements}

The authors thank our ADAMANT colleagues, particularly V. Tountopoulos, N. Mitrou, L. Cuthbert, Y. Wang, R. Futiem, M. Sentris, and M. Senis.

\section{References}

[1] A. L. G. Hayzelden, J. Bigham (eds.), Software Agents for Future Communications Systems, Springer-Verlag, 1999.

[2] IST SHUFFLE Project, IST-1999-11014, Deliverable D5, "Report on agent control of resources", Nov 2000.

[3] Y. Wang, L. Cuthbert, F. Mullany, et al., "Exploring agentbased wireless business models and decision support applications in an airport environment", Journal of Telecommunications and Information Technology, pp. 5764, no. 3, 2004.

[4] WWI Ambient Networks Project, IST Project Number 507134, http://www.ambient-networks.org/.

[5] Wireless World Research Forum, "The Book of Visions 2001: Visions of the Wireless World", http://www.wwrf.org/general_info/bookofvisions/bov.html, 2001.

[6] J. Ferber, Multi-Agent Systems: An Introduction to Distributed Artificial Intelligence, Addison-Wesley, 1998.

[7] 3GPP TSG SA, "IP Multimedia Subsystem (IMS); Stage 2 (Release 5)," 3GPP TS 23.228, v5.12.0, Mar 2004.

[8] G. Vincent, "Learning from i-mode", IEE Review, vol. 47, no. 6, pp. 13-18, Nov. 2001.

[9] Ofcom, "Spectrum Framework Review", consultation document, http://www.ofcom.org.uk/consultations/, Nov. 2004.

[10] P. Kolodzy et al., Spectrum Policy Task Force Report, FCC, ET docket no. 02-135, Nov. 2002

[11] P. Klemperer, "Auction Theory: A Guide to the Literature", Journal of Economic Surveys, vol.13(3), pp.227-286, July 1999.

[12] Qualcomm, "The Economics of Wireless Data", white paper, March 2001.

[13] COST 231 Final Report "Digital mobile radio towards future generation systems," http://www.lx.it.pt/cost231/

[14] TR $101112 \mathrm{v} 3.2 .0$, "Selection procedures for the choice of radio transmission technologies for the UMTS (UMTS 30.03 version 3.2.0), ’ Jan. 2001.

[15] A. M. Law, W. D. Kelton, Simulation Modeling and Analysis, Third Edition, McGraw-Hill, 2000.

[16] C. Courcoubertis, R. Weber, Pricing Communication Networks; Economics, Technology and Modelling, John Wiley and Sons, 2003. 